\title{
Atrial strain: an important addition to the armamentarium of non-invasively assessing Fontan function?
}

\author{
Arend D. J. Ten Harkel ${ }^{1}$. Annelies E. Van der Hulst ${ }^{2}$ \\ Published online: 10 July 2021 \\ (c) The Author(s), under exclusive licence to Springer Nature B.V. 2021
}

Patients with univentricular hearts are being palliated mostly with the total cavopulmonary connection, either by an extracardiac conduit (ECC) or an intra-atrial lateral tunnel (ILT). Despite the excellent results with regard to volume unloading and oxygen saturation, Fontan patients on the long-term are at high risk of reduced exercise capacity, clinical deterioration and premature death [1]. Although many Fontan patients deteriorate over time it is difficult to identify which patients are at most risk and much effort has therefore been undertaken to search for predictive variables. Contributing factors leading to adverse outcomes are multiple and include diastolic and systolic ventricular dysfunction, rhythm disturbances including brady- and tachy-arrhythmias, atrioventricular valve regurgitation, increased pulmonary artery pressure, complex anatomy leading to inflow or outflow obstructions and thrombo-embolic events [2]. Currently, the role of atrial function in the (failing) Fontan circulation is being increasingly studied. The present study of Veldtman et al. contributes importantly to this expanding knowledge [3].

In the Fontan circulation, the maximum cardiac output is often limited to the ventricular preload. As a result, in Fontan patients with impaired ventricular diastolic function, which is frequently encountered, the atria may play an important role in maintaining adequate ventricular filling [4]. Atrial function can be divided in atrial reservoir function (during ventricular systole), atria conduit function (during

This comment refers to the article available online at https://doi. org/10.1007/s10554-021-02298-w.

Arend D. J. Ten Harkel

a.d.j.ten_harkel@lumc.nl

1 Department of Pediatric Cardiology, Willem Alexander Children's Hospital, Leiden University Medical Center, Leiden, The Netherlands

2 Department of Pediatric Cardiology, Emma Children's Hospital, Amsterdam University Medical Center,

Amsterdam, The Netherlands early diastole) and atrial contraction function (during late diastole). Novel post-processing applications after echocardiography as well as cardiac MRI have enabled non-invasive assessment of these different components of atrial function using strain, as explained in the present issue by Veldtman et al. [3]. In healthy adults, atrial function as assessed by echocardiographic strain has been shown to relate to exercise capacity [5].

In children, Khoo et al. [6] studied the development of the various atrial functions in healthy hearts as well as in univentricular hearts at the age of 0 to 6.5 years using echocardiographic strain measurements. In healthy growing children, atrial reservoir function increased with age, whereas the contribution of atrial contraction function became less important over time. In single ventricle patients, during the same period in different stages of their palliative trajectory (pre-Glenn, post-Glenn, pre-Fontan completion and postFontan completion) the increase in atrial reservoir function was absent, and this observed difference between normal children and children with single ventricles increased over time, already being significantly different before any surgical intervention. In contrast, the atrial contractile function was consistently higher in the patients as compared to controls in all stages. This finding suggests that adequate diastolic function in the Fontan patient relies more on atrial contraction function for ventricular filling as compared to normal controls. This is especially of importance in Fontan patients without adequate sinus rhythm, which frequently occurs.

Various authors studied the relation among atrial strain measurements in Fontan patients and clinical relevant outcome parameters, yielding conflicting results. Van der Ven et al. [7] assessed the role of atrial function, as assessed with atrial feature tracking strain with cardiac MRI, on exercise capacity and clinical events in 95 Fontan patients. In this study, atrial function did not predict exercise capacity or events during follow up. In contrast, two other recent studies did find correlations between reduced echocardiographic 
strain and diminished exercise capacity [8] and adverse clinical outcomes [9] in Fontan patients.

In the present issue, Veldtman et al. [3] evaluated atrial function in Fontan patients, and compared it to invasively measured hemodynamic parameters. As in other studies, global atrial strain was lower as compared to normal controls. In addition, in the present study, all three components of atrial function (atrial reservoir, atrial conduit and atrial contractile function) were reduced. Systemic ventricular filling pressures were not correlated to any of the atrial function parameters, although global atrial conduit strain was indeed correlated to the E/A ratio of the $\mathrm{AV}$ valve, which was on its turn correlated to global longitudinal strain of the systemic ventricle. This finding suggests an important coupling among atrial function and diastolic ventricular function, influencing atrial flow mechanics as well as systolic ventricular performance. In the study of Peck et al. [9] atrial strain was not correlated to systolic end diastolic pressure, as found in the article of Veldtman et al. [3], but reduced atrial reservoir strain was indeed related to adverse clinical outcomes.

Thus, unexplained discrepancies remain among the various reports on atrial strain and function in Fontan patients, as well as its relation to clinical relevant outcome parameters. Several major differences among these studies may be noted. Van der Ven et al. [7] observed differences in atrial reservoir function among Fontan patient with ECC versus patients with ILT, whereas this difference was not accounted for or reported in other reports. In addition to these surgical differences, Fontan patients may greatly vary in ventricular morphology and intra- and extracardiac anatomy. Larger studies are needed to comprehensively address these heterogeneities. Another important discrepancy among the mentioned atrial strain studies concerns the atrial region of interest; In the study of Khoo et al. [6], atrial strain was assessed by tracing the 'dominant' atrium (i.e. the atrium confined to the single ventricle), whereas the other studies [7-9] traced the entire common atrium. Veldtman et al. [3] measured both; atrial strain among the common atrium (referred to as global atrial strain) as well as 'regional atrial strain', referring to the left atrial wall. Importantly, no correlation among global atrial strain and regional atrial strain were noted. In our opinion, the latter finding underscores the importance of additional investigations with the objective of reaching consensus on the method and role of atrial strain measurements during follow up of this vulnerable patient group. Since both atria in the Fontan circulation are closely related to each other with an unrestricted atrial septal defect to our opinion at least global atrial strain should be measured.

In conclusion, the hemodynamics and clinical relevance underlying the role of atrial function and atrial strain in Fontan patients are as yet incompletely understood. Preload, atrial scarring, systolic and diastolic ventricular function as well as pulmonary vascular resistance all may play a role and methodological consensus regarding the strain measurements are needed. However, using echocardiography, atrial strain measurement is a feasible, readily available method that may prove to be a valuable additional tool in risk stratification of Fontan patients in the near future.

\section{References}

1. Schwartz I, McCracken CE, Petit CJ, Sachdeva R (2018) Late outcomes after the Fontan procedure in patients with single ventricle: a meta-analysis. Heart 104:1508-1514

2. Poh CL, d'Udekem Y (2018) Life after surviving fontan surgery: a meta-analysis of the incidence and predictors of late death. Heart Lung Circ 27:552-559

3. Veldtman G, Possner M, Mohty D et al (2021) Atrial function in the Fontan circulation: comparison with invasively assessed systemic ventricular filling pressure. Int J Cardiovasc Imag. https:// doi.org/10.1007/s10554-021-02298-w

4. Li SJ, Wong SJ, Cheung YF (2014) Atrial and ventricular mechanics in patients after Fontan-type procedures: atriopulmonary connection versus extracardiac conduit. J Am Soc Echocardiogr 27:666-674

5. Leite L, Mendes SL, Baptista R et al (2017) Left atrial mechanics strongly predict functional capacity assessed by cardiopulmonary exercise testing in subjects without structural heart disease. Int $\mathbf{J}$ Cardiovasc Imaging 33:635-642

6. Khoo NS, Smallhorn JF, Kaneko S, Kutty S, Altamirano L, Tham EB (2013) The assessment of atrial function in single ventricle hearts from birth to Fontan: a speckle-tracking study by using strain and strain rate. J Am Soc Echocardiogr 26:756-764

7. van der Ven JPG, Alsaied T, Juggan S et al (2020) Atrial function in Fontan patients assessed by CMR: relation with exercise capacity and long-term outcomes. Int J Cardiol 312:56-61

8. Rato J, Mendes SC, Sousa A, Lemos M, Martins DS, Anjos R (2020) The influence of atrial strain on functional capacity in patients with the fontan circulation. Pediatr Cardiol 41:1730-1738

9. Peck D, Alsaied T, Pradhan S, Hill G (2021) Atrial reservoir strain is associated with decreased cardiac index and adverse outcomes post Fontan operation. Pediatr Cardiol 42:307-314

Publisher's Note Springer Nature remains neutral with regard to jurisdictional claims in published maps and institutional affiliations. 